\title{
On Approximation Hardness of the Minimum 2SAT-DELETION Problem
}

\author{
Miroslav Chlebík ${ }^{1}$ and Janka Chlebíkováa ${ }^{2 \star}$ \\ 1 MPI for Mathematics in the Sciences, D-04103 Leipzig, Germany, \\ chlebik@mis.mpg.de \\ 2 Department of Informatics Education, Faculty of Mathematics, Physics and \\ Informatics, Comenius University, Mlynská dolina, 84248 Bratislava, Slovakia, \\ chlebikj@dcs.fmph.uniba.sk
}

\begin{abstract}
The Minimum 2SAT-Deletion problem is to delete the minimum number of clauses in a $2 \mathrm{SAT}$ instance to make it satisfiable. It is one of the prototypes in the approximability hierarchy of minimization problems [8], and its approximability is largely open. We prove a lower approximation bound of $8 \sqrt{5}-15 \approx 2.88854$, improving the previous bound of $10 \sqrt{5}-21 \approx 1.36067$ by Dinur and Safra [5]. For highly restricted instances with exactly 4 occurrences of every variable we provide a lower bound of $\frac{3}{2}$. Both inapproximability results apply to instances with no mixed clauses (the literals in every clause are both either negated, or unnegated).

We further prove that any $k$-approximation algorithm for Minimum 2SAT-DELETION polynomially reduces to a $\left(2-\frac{2}{k+1}\right)$-approximation algorithm for the Minimum VerTex Cover problem.

One ingredient of these improvements is our proof that for the Minimum VERTEX COVER problem restricted to graphs with a perfect matching its threshold on polynomial time approximability is the same as for the general Minimum VerTex Cover problem. This improves also on results of Chen and Kanj [3].
\end{abstract}

Keywords: vertex cover, perfect matching, satisfiability, combinatorial optimization problems, approximation algorithm, inapproximability, NemhauserTrotter Theorem

\section{Introduction}

The proof of the PCP Theorem and subsequent improvements in PCP constructions have led for many optimization problems to optimal bounds on their efficient approximability (unless $\mathrm{P}=\mathrm{NP}$ ). However, in spite of a great deal of efforts, for several fundamental problems the tight bound on their approximability by a polynomial time algorithm is left open, for example: Is there a polynomial time approximation algorithm for the MinIMUM VERTEX COVER problem

* The author has been partially supported by the grant VEGA 1/0131/03. 
whose approximation factor is bounded above by a constant less than 2? Is there a polynomial time algorithm that approximates the MINIMUM 2SAT-DELETION problem to within a constant factor?

Recall that the task in the Minimum 2SAT-Deletion problem (shortly, Min-2SAT-DeLETion) is to delete the minimum number of clauses in a 2SAT instance to make it satisfiable. The problem is important as one of the prototypes in a complete classification of the approximability of minimization problems derived from Boolean constraint satisfaction [8].

Currently, the best lower bound on polynomial time approximability is the same for both problems, namely $10 \sqrt{5}-21 \approx 1.36067$, due to Dinur and Safra [5]. For the Min-2SAT-DELETION problem we can improve on this bound, even on highly restricted instances where every variable occurs in very small number of clauses. We show how two questions mentioned above are related to one another: the affirmative answer to the second question would imply the affirmative answer to the first one.

\section{Our Results}

We concentrate mainly on the Minimum VerTex Cover problem (Min-VC) on graphs with a perfect matching (MiN-VC-PM). It turns out that the problems to solve Min-VC exactly, and to approximate Min-VC within a factor on general graphs, reduce to the corresponding problems on graphs with a perfect matching. In particular, the threshold on polynomial time approximability of MIN-VC is the same as for Min-VC-PM. Moreover, we observe that the NP-hard gap results of Dinur and Safra [5] for Min-VC apply to Min-VC-PM as well. Using this fact and the powerful reduction from Min-VC-PM to Min-2SAT-DeLETION we can improve inapproximability results for Min-2SAT-DELETION. We prove that it is NP-hard to approximate MIN-2SAT-DELETION to within any constant factor less than $8 \sqrt{5}-15 \approx 2.88854$. We provide interesting lower bound also for small occurrence instances: it is NP-hard to approximate Min-2SAT-DeLETION to within any constant factor less than $\frac{3}{2}$ on instances with exactly 4 occurrences of every variable. Both inapproximability results apply to instances with no mixed clauses (i.e., only clauses $x \vee y, \bar{x} \vee \bar{y}$, with $x$ and $y$ distinct variables, are allowed).

We further prove that the existence of a polynomial time approximation algorithm for MIN-2SAT-DELETION with a constant factor $k, k \in(1, \infty)$, would imply the existence of a polynomial time $\left(2-\frac{2}{k+1}\right)$-approximation algorithm for Min-VC. Even slight improvement to $o\left(\frac{\log n}{\log \log n}\right)$ of the approximation factor for Min-2SAT-Deletion will improve on currently the best polynomial time approximation factor $\left(2-\frac{\log \log n}{2 \log n}\right)$ for MIN-VC ([1], [12]). Moreover, we observe that the existence of an algorithm for MAX-2SAT that for some $t>\frac{1}{2}$ for every instance finds an assignment satisfying at least a fraction $\left(1-O\left(\varepsilon^{t}\right)\right)$ of clauses, assuming that optimal assignment satisfies a fraction $(1-\varepsilon)$ of them, will improve on known results for MiN-VC in sparse graphs. 


\section{Preliminaries}

Let us start with the formulation of problems we will deal with and some known results about them.

Definition 1. Consider a set of clauses $C_{1}, C_{2}, \ldots, C_{N}$ with each clause $C_{i}$ of the form $l_{1} \vee l_{2}$ and nonnegative weights $w_{i}$. Each literal $l_{j}$ is either one of Boolean variables $x_{1}, x_{2}, \ldots, x_{n}$, or its negation. A common variant of the above is that all weights are the same but clauses can be repeated. The goal of the MAX2SAT problem is to assign Boolean values 0 and 1 to variables $x_{1}, x_{2}, \ldots, x_{n}$ so that the total weight of the satisfied clauses is maximized. For the complementary problem of minimum unsatisfiability, Min-2SAT-DELETION (called also Min2CNF-DELETION by some authors), the goal is to minimize the total weight of unsatisfied clauses over all assignments.

While MAX-2SAT is approximable within 1.0638 [11] and it is NP-hard to approximate within 1.0476 [6], the approximability of the Min-2SAT-DELETION problem is still widely open problem. The problem is known to be approximable in polynomial time to within polylogarithmic factor $O(\log n \log \log n)$ (Klein et al. [9]) and, on the other hand, to be NP-hard to approximate to within $1.36067[5]$.

Let $G=(V, E)$ be a simple graph. For a set of vertices $U \subseteq V$, let $\Gamma(U):=$ $\{v \in V: \exists u \in U$ such that $\{u, v\} \in E\}$ stand for the set of its neighbors, and $G[U]$ denote the subgraph of $G$ induced by $U$.

\section{Minimum Vertex Cover}

Instance: A simple graph $G=(V, E)$.

Feasible solution: A vertex cover $C$ for $G$, i.e., a subset $C \subseteq V$ such that for each $e \in E, e \cap C \neq \emptyset$.

Objective function: The cardinality $|C|$ of the vertex cover $C$.

Let $v c(G)$ stand for the cardinality of the minimum vertex cover for $G$. Similarly, a half-integral vertex cover for $G=(V, E)$ is a function $x: V \rightarrow\left\{0, \frac{1}{2}, 1\right\}$ satisfying edge constraints $x(u)+x(v) \geq 1$ for each edge $\{u, v\} \in E$. Let $v c_{*}(G)$ stand for the cardinality of the minimum half-integral vertex cover for $G$, i.e., the minimum of $w(x):=\sum_{u \in V} x(u)$ over all half-integral vertex covers $x$.

Clearly, $v c_{*}(G) \leq v c(G)$, as for any vertex cover $C$ its indicator function $x^{C}$ is also a half-integral vertex cover with $w\left(x^{C}\right)=|C|$. Further, $v c_{*}(G) \leq \frac{1}{2}|V|$, as the function $x \equiv \frac{1}{2}$ on $V$ is always the half-integral vertex cover for $G$.

The following theorem of Nemhauser and Trotter [13] is of great importance for many problems related to MiN-VC problems. It allows to reduce the problem to instances, where the value of a minimum vertex cover is at least $\frac{1}{2}|V|$.

Nemhauser-Trotter Theorem (NT-Theorem). There exists a polynomial time algorithm that partitions the vertex set $V$ of any graph $G$ into three subsets $V_{0}, V_{1}, V_{\frac{1}{2}}$ with no edges between $V_{0}$ and $V_{\frac{1}{2}}$ or within $V_{0}$ such that

(i) $v c\left(G\left[V_{\frac{1}{2}}\right]\right) \geq v c_{*}\left(G\left[V_{\frac{1}{2}}\right]\right)=\frac{1}{2}\left|V_{\frac{1}{2}}\right|$; and 
(ii) there exists some minimum vertex cover $C$ for $G$ such that $V_{1} \subseteq C \subseteq V_{1} \cup V_{\frac{1}{2}}$ and $C \cap V_{\frac{1}{2}}$ is a minimum vertex cover for $G\left[V_{\frac{1}{2}}\right]$.

In what follows we define for a graph $G$ its bipartite version $G^{b}$ and observe that the minimum half-integral vertex covers for $G$ are generated by minimum vertex covers for the corresponding bipartite graph $G^{b}$.

Definition 2. For a graph $G=(V, E)$ we define its bipartite version $G^{b}=$ $\left(V^{b}, E^{b}\right)$, as follows: there are two copies $u^{L}$ and $u^{R}$ of each vertex $u \in V$ in $G^{b}, V^{L}:=\left\{u^{L}: u \in V\right\}, V^{R}:=\left\{u^{R}: u \in V\right\}$, and $V^{b}:=V^{L} \cup V^{R}$. Each edge $\{u, v\} \in E$ of $G$ creates two edges in $G^{b}$, namely $\left\{u^{L}, v^{R}\right\}$ and $\left\{v^{L}, u^{R}\right\}$. Hence $E^{b}:=\left\{\left\{u^{L}, v^{R}\right\},\left\{v^{L}, u^{R}\right\}:\{u, v\} \in E\right\}$. For $U \subseteq V$ we use also $U^{L}, U^{R}$, and $U^{b}:=U^{L} \cup U^{R}$ for the corresponding sets of vertices.

For any set $C \subseteq V^{\mathrm{L}} \cup V^{\mathrm{R}}$ we associate a map $x_{C}: V \rightarrow\left\{0, \frac{1}{2}, 1\right\}$ in the following way: $x_{C}(\bar{u})=\frac{1}{2}\left|C \cap\left\{u^{\mathrm{L}}, u^{\mathrm{R}}\right\}\right|$ for any $u \in V$. Clearly $w\left(x_{C}\right)=\frac{1}{2}|C|$ for any $C \subseteq V^{\mathrm{L}} \cup V^{\mathrm{R}}$.

Lemma 1. (i) If $C$ is a vertex cover for $G^{b}$ then $x_{C}$ is a half-integral vertex cover for $G$ of weight $\frac{1}{2}|C|$. In particular, $v c_{*}(G) \leq \frac{1}{2} v c\left(G^{b}\right)$.

(ii) If $x: V \rightarrow\left\{0, \frac{1}{2}, 1\right\}$ is a half-integral vertex cover for $G$ then there is a vertex cover $C$ for $G^{b}$ such that $x_{C}=x$. Hence $\frac{1}{2} v c\left(G^{b}\right) \leq v c_{*}(G)$.

(iii) $v c_{*}(G)=\frac{1}{2} v c\left(G^{b}\right)$

Proof. (i) being obvious, it is sufficient to show (ii) and the rest will trivially follow. Given $x: V \rightarrow\left\{0, \frac{1}{2}, 1\right\}$ as required, let $V_{i}^{x}:=\{u \in V: x(u)=i\}$ for $i \in\left\{0, \frac{1}{2}, 1\right\}$. Obviously, there are no edges between $V_{0}^{x}$ and $V_{\frac{1}{2}}^{x}$ or within $V_{0}^{x}$. It follows that a set $C$ defined by $C:=\bigcup_{u \in V_{1}^{x}}\left\{u^{\mathrm{L}}, u^{\mathrm{R}}\right\} \cup \bigcup_{u \in V_{\frac{1}{2}}^{x}}\left\{u^{\mathrm{L}}\right\}$ is a vertex cover for $G^{b}$ such that $x_{C}=x$.

\section{Min-VC for Graphs with Perfect Matching}

This section concentrates on the Minimum VerTex COVER problem on graphs with a perfect matching. We will show that the problems to solve Min-VC exactly and to approximate MiN-VC to within a factor in general graphs reduce to the corresponding MiN-VC problems on graphs with a perfect matching.

Definition 3. Given a graph $G=(V, E)$, let $\widetilde{G}=(\widetilde{V}, \widetilde{E})$ be a 2-padding of $G$ with the same vertex set as $G^{b}$ (Definition 2 ), i.e., $\widetilde{V}=V^{b}$ and

$$
\widetilde{E}=E^{b} \cup\left\{\left\{u^{L}, v^{L}\right\},\left\{u^{R}, v^{R}\right\}:\{u, v\} \in E\right\} .
$$

Theorem 1. The threshold on polynomial time approximability of the MiN-VC problem is the same as the one for the MIN-VC-PM problem. 
Proof. Nemhauser-Trotter Theorem reduces the Min-VC problem for $G=(V, E)$ in approximation preserving way to its induced subgraph $G\left[V_{\frac{1}{2}}\right]$ with the property $v c\left(G\left[V_{\frac{1}{2}}\right]\right) \geq v c_{*}\left(G\left[V_{\frac{1}{2}}\right]\right)=\frac{1}{2}\left|V_{\frac{1}{2}}\right|$.

Let $\widetilde{G}$ be a 2-padding of $G$. Obviously if $C \subseteq V$ is a vertex cover in $G$ then $\widetilde{C}:=\cup_{u \in C}\left\{u^{\mathrm{L}}, u^{\mathrm{R}}\right\}$ is a vertex cover in a $\widetilde{G}$. Moreover, every minimal (on inclusion) vertex cover of $\widetilde{G}$ is of the form above for some vertex cover $C$ of $G$.

Using Lemma 1(iii) and König-Egerváry Theorem (see, e.g., [14]) the fact $v c_{*}\left(G\left[V_{\frac{1}{2}}\right]\right)=\frac{1}{2}\left|V_{\frac{1}{2}}\right|$ applied to $G\left[V_{\frac{1}{2}}\right]$ implies that $\left(G\left[V_{\frac{1}{2}}\right]\right)^{b}$ has a perfect matching, which in turn implies that $\widetilde{G\left[V_{\frac{1}{2}}\right]}$ has a perfect matching. But problems Min-VC for $G\left[V_{\frac{1}{2}}\right]$ and $\widetilde{G\left[V_{\frac{1}{2}}\right]}$ are essentially equivalent. Hence the Min-VC problem reduces to the MIN-VC-PM problem.

We can show explicitly that the NP-hard gap result of Dinur and Safra [5] applies to the Min-VC-PM problem as well. As their construction is rather involved, we don't reproduce it and refer the reader to [5] for more details. Consider one of weighted graphs they generate, then there is clearly a perfect matching within every long-code block by connecting each subset to its complement. However, after standard multiplication the vertices according to (positive rational) weights to get the unweighted version of their NP-hard gap result, such matching is no longer perfect. The question of whether there is a perfect matching in this graph reduces to the one of whether there is a perfect matching in the Kneser graph, the main building block of their construction. But it is well known even for every connected vertex transitive graph that it has a perfect matching if it has even number of vertices, and it has a nearly perfect matching if the number of vertices is odd (see [10]). Therefore their NP-hard gap result applies to Min-VC-PM as well. It can be stated as follows

Theorem 2. (Dinur and Safra) Let $p, q$ be constants such that $\frac{3-\sqrt{5}}{2}>p>$ $q>\max \left\{p^{2}, 4 p^{3}-3 p^{4}\right\}$. It is NP-hard for graphs $G=(V, E)$ with a perfect matching to distinguish between the following two cases: $v c(G)<(1-p)|V|$, or $v c(G)>(1-q)|V|$.

The Theorem 1 is true for general graphs, or for any class $\mathcal{G}$ of graphs that is closed on operation of taking a 2-padding of an induced subgraph. If the class $\mathcal{G}$ of graphs does not have this property, but it is at least closed on the operation of taking an induced subgraph (e.g., $\mathcal{G}=\{$ graphs with the maximum degree $\leq B\}$, or $\mathcal{G}=\{$ everywhere $B$-sparse graphs, for some constant $B\}$ ), we can conclude that Min-VC restricted to $\mathcal{G}$ is as hard to approximate as Min-VC restricted to $\mathcal{G}^{\prime}$, where $\mathcal{G}^{\prime}$ consists of graphs $G=(V, E)$ from $\mathcal{G}$ with $v c_{*}(G)=\frac{1}{2}|V|$. In such classes $\mathcal{G}$ we do not know if Min-VC-PM is as hard to approximate as Min-VC, or easier. 


\section{Application to 2SAT Problems}

In what follows we relate the Minimum Vertex Cover problem to MaX2SAT and similar problems. Similarly as in [3] we use the following reduction from Min-VC-PM to MAX-2SAT.

2SAT-construction. Let $G=(V, E)$ be an instance of Min-VC-PM and let $M$ be a fixed perfect matching in $G$. Define an instance $\mathcal{F}(G, M)$ of 2SAT as follows: the Boolean variable set $X_{V}:=\left\{x_{u}: u \in V\right\}$ and

$$
\mathcal{F}(G, M)=\bigcup_{\{u, v\} \in E}\left\{\left(x_{u} \vee x_{v}\right)\right\} \cup \bigcup_{\{u, v\} \in M}\left\{\left(\bar{x}_{u} \vee \bar{x}_{v}\right)\right\}
$$

Hence $\mathcal{F}(G, M)$ consists of $N:=|E|+\frac{1}{2}|V|$ clauses. All the clauses have exactly 2 (different) literals and are non-mixed, i.e., none of the clauses has both negated and unnegated literals. This variant of 2SAT is sometimes referred to as E2-NMSAT (NM stands for non-mixed clauses). Obviously the instance $\mathcal{F}:=\mathcal{F}(G, M)$ of E2-NM-SAT defined above has three additional properties:

(P1) clauses are not repeated,

(P2) each variable appears exactly once as negated; and

(P3) if $(\bar{a} \vee \bar{b}) \in \mathcal{F}$ for variables $a$ and $b$, then $(a \vee b) \in \mathcal{F}$ as well.

Let unsat $(\sigma)$ be the number of clauses of $\mathcal{F}(G, M)$ that are unsatisfied by an assignment $\sigma: X_{V} \rightarrow\{0,1\}$, and $\operatorname{OPT}(\mathcal{F}(G, M))$ be the minimum of unsat $(\sigma)$ over all assignments $\sigma: X_{V} \rightarrow\{0,1\}$. Then the following lemma holds

Lemma 2. Let $M$ be a perfect matching in a graph $G=(V, E)$ and the collection of clauses $\mathcal{F}(G, M)$ of Boolean variables $X_{V}$ be according to the construction above. Then

(i) $\operatorname{OPT}(\mathcal{F}(G, M))=v c(G)-\frac{1}{2}|V|$,

(ii) From any assignment $\sigma: X_{V} \rightarrow\{0,1\}$ a vertex cover $C$ for $G$ of cardinality at most $\left(\frac{1}{2}|V|+\operatorname{unsat}(\sigma)\right)$ can be constructed in time $O(|E|)$.

Proof. If $C$ is a vertex cover in $G$, let $\sigma_{C}: X_{V} \rightarrow\{0,1\}$ denote the following assignment: $\sigma_{C}\left(x_{u}\right)=1$ iff $u \in C$. The fact that $C$ was a vertex cover means exactly that all clauses with unnegated variables are satisfied by $\sigma_{C}$. Such assignment to variables will be called standard. Clearly, standard assignments $\sigma: X_{V} \rightarrow\{0,1\}$ are in one-to-one correspondence with vertex covers in $G$.

It is clear that for a standard assignment $\sigma_{C}$, the edges in a perfect matching $M$ are partitioned into two sets $M_{1}$ and $M_{2}$ such that each edge $\{u, v\}$ in $M_{i}$ has exactly $i$ endpoints in $C, i=1,2$. Thus $|C|=\left|M_{1}\right|+2 \cdot\left|M_{2}\right|$, and clearly unsat $\left(\sigma_{C}\right)=\left|M_{2}\right|=|C|-\left(\left|M_{1}\right|+\left|M_{2}\right|\right)=|C|-\frac{1}{2}|V|$. Consequently, $\operatorname{OPT}(\mathcal{F}(G, M)) \leq v c(G)-\frac{1}{2}|V|$.

Let now an assignment $\sigma: X_{V} \rightarrow\{0,1\}$ be given. We can modify $\sigma$ (in time $O(|E|))$ to the standard assignment $\sigma_{C}$ such that unsat $\left(\sigma_{C}\right) \leq \operatorname{unsat}(\sigma)$. In 
another words, we can provide in time $O(|E|)$ a vertex cover $C$ with unsat $\left(\sigma_{C}\right)=$ $|C|-\frac{1}{2}|V| \leq \operatorname{unsat}(\sigma)$.

We will process edges $\{u, v\} \in E$, one after another, as follows: If $\sigma\left(x_{u}\right)=$ $\sigma\left(x_{v}\right)=0$ for an edge $\{u, v\} \in E$, then we modify $\sigma$ at exactly one endpoint of $\{u, v\}$, say $u$, setting $\sigma\left(x_{u}\right)=1$ instead. This change does not increase the value unsat $(\sigma)$ : the unsatisfied clause $\left(x_{u} \vee x_{v}\right)$ becomes satisfied, and at most one satisfied clause can become unsatisfied, namely the one containing the literal $\bar{x}_{u}$. Having this done, one after another, for all edges $\{u, v\} \in E$, the resulting assignment will be standard, hence of the form $\sigma_{C}$ for a vertex cover $C$ of $G$, and unsat $\left(\sigma_{C}\right) \leq \operatorname{unsat}(\sigma)$.

In particular, it follows (taking $\sigma$ with unsat $(\sigma)=\operatorname{OPT}(\mathcal{F}(G, M)$ ) that $v c(G)-\frac{1}{2}|V|=\operatorname{OPT}(\mathcal{F}(G, M))$.

Using the 2SAT-construction above from MiN-VC-PM to special instances of Min-2SAT-DELETION we can obtain from Theorem 2

Theorem 3. Let $p, q$ be constants such that $\frac{3-\sqrt{5}}{2}>p>q>\max \left\{p^{2}, 4 p^{3}-\right.$ $\left.3 p^{4}\right\}$. It is NP-hard for instances $\mathcal{F}$ of MiN-2SAT-DELETION to distinguish between two cases: $\mathrm{OPT}(\mathcal{F})<\left(\frac{1}{2}-p\right)|X|$ or $\mathrm{OPT}(\mathcal{F})>\left(\frac{1}{2}-q\right)|X|$, where $X$ is the set of Boolean variables of $\mathcal{F}$. Consequently, it is NP-hard to approximate the MIN-2SAT-DELETION problem to within any constant approximation factor smaller than $8 \sqrt{5}-15 \approx 2.88854$. The same NP-hardness result applies to instances with no mixed clauses, and satisfying conditions $(\mathrm{P} 1)-(\mathrm{P} 3)$.

Proof. The $\left(\left(\frac{1}{2}-p\right)|X|,\left(\frac{1}{2}-q\right)|X|\right)$-gap result follows directly from the reduction $G \mapsto \mathcal{F}(G, M)$ using Theorem 1 and Lemma 2. Hence inapproximability to within $\frac{1-2 q}{1-2 p}$ follows, for any $p, q$ satisfying our assumptions. Notice that for $p \in\left(\frac{1}{3}, 1\right), \max \left\{p^{2}, 4 p^{3}-3 p^{4}\right\}=4 p^{3}-3 p^{4}$, and as $q$ can approach $4 p^{3}-3 p^{4}$ (from above) and $p$ can approach $\frac{3-\sqrt{5}}{2}$ (from below), $\frac{1-2 q}{1-2 p}$ can approach $8 \sqrt{5}-15$ (from below). Hence NP-hardness to approximate the problem to within any constant factor smaller than $8 \sqrt{5}-15$ follows.

Assume now that we have an approximation algorithm $\mathscr{A}$ for the Min-2SATDELETION problem. The above polynomial time 2SAT-construction suggests an approximation algorithm (based on $\mathscr{A}$ ) for MiN-VC-PM and, consequently, for the Min-VC problem.

Theorem 4. Given an algorithm that approximates the solution of the MiN2SAT-DELETION problem within approximation factor $f(n, N) \geq 1$ on instances with $n$ variables and $N$ clauses, all non-mixed and satisfying $(\mathrm{P} 1)-(\mathrm{P} 3)$. (Here $f: \mathbb{N}_{+}^{2} \rightarrow\langle 1, \infty)$ is a function separately nondecreasing in every variable.) It can be reduced to the one that approximates MiN-VC-PM (respectively, MIN-VC) on instances with $n$ vertices and $m$ edges within $2-\frac{2}{f\left(n, m+\frac{n}{2}\right)+1}$ (respectively, $\left.2-\frac{2}{f(2 n, 4 m+n)+1}\right)$.

Proof. Let $G=(V, E)$ be a graph with $n:=|V|$ and $m:=|E|$. We can assume that $G$ has a perfect matching. Otherwise we can work with a graph $\widetilde{G}\left[V_{\frac{1}{2}}\right]$, that 
is a graph with $n^{\prime} \leq 2 n$ vertices, $m^{\prime} \leq 4 m$ edges, and with a perfect matching. Let $\mathscr{A}$ be an $f$-approximation algorithm for Min-2SAT-DELETION, and consider the following algorithm:

Step 1: Construct a perfect matching $M$ in $G$,

Step 2: construct the corresponding instance $\mathcal{F}(G, M)$ of the Min-2SAT-DeLETION problem, with $n$ variables and $N:=m+\frac{n}{2}$ clauses,

Step 3: applying the algorithm $\mathscr{A}$ to $\mathcal{F}(G, M)$ construct an assignment $\sigma$ : $X_{V} \rightarrow\{0,1\}$ that approximates the optimal solution for $\mathcal{F}(G, M)$ within $f:=f(n, N)$,

Step 4: construct a vertex cover $C$ in $G$ of cardinality at most $\left(\frac{1}{2}|V|+\operatorname{unsat}(\sigma)\right)$, according to Lemma 2,

Step 5: return a vertex cover $C$ of $G$.

Our aim is to show that the algorithm returns a vertex cover $C$ of $G$, with the property $|C| \leq v c(G) \cdot\left(2-\frac{2}{f+1}\right)$. The assumptions on $\mathscr{A}$ guarantee that the assignment $\sigma$ provided in Step 3 satisfies

$$
\operatorname{unsat}(\sigma) \leq \operatorname{OPT}(\mathcal{F}(G, M)) \cdot f \text {. }
$$

If unsat $(\sigma) \geq \frac{1}{2}|V|$, we conclude from (1) and from Lemma 2 that

$$
v c(G)=\frac{1}{2}|V|+\operatorname{OPT}(\mathcal{F}(G, M)) \geq \frac{1}{2}|V|+\frac{1}{2} \frac{|V|}{f} .
$$

As clearly $|C| \leq|V|, \frac{|C|}{v c(G)} \leq 2-\frac{2}{f+1}$ easily follows.

If unsat $(\sigma)=\frac{1}{2}|V| \cdot t$ for $t \in\langle 0,1\rangle$, we conclude similarly that

$$
v c(G) \geq \frac{1}{2}|V|+\frac{1}{2}|V| \frac{t}{f},
$$

but now we use better estimate on $|C|$ from Step $4,|C| \leq \frac{1}{2}|V|+\frac{1}{2}|V| t$. Hence

$$
\frac{|C|}{v c(G)} \leq \frac{1+t}{1+\frac{t}{f}}=\frac{(1+t) f}{f+t} .
$$

The right hand side as a function of $t \in\langle 0,1\rangle$ achieves its maximum at $t=1$ (we use that $f \geq 1$ for this argument), hence

$$
\frac{|C|}{v c(G)} \leq \frac{2 f}{f+1}=2-\frac{2}{f+1} .
$$

Corollary 1. To approximate MIN-2SAT-DELETION within a constant $k$ is at least as difficult (up to polynomial reduction between problems) as to approximate MiN-VC within a factor $2-\frac{2}{k+1}$. 
If we apply Theorem 4 to the $O(\log n \log \log n)$-approximation algorithm given for Min-2SAT-DeLETion by Klein et al. [9], we obtain an algorithm for Min-VC with the approximation factor $\left(2-\frac{1}{c \log n \log \log n}\right)$. An improvement to $o\left(\frac{\log n}{\log \log n}\right)$ of the above factor for Min-2SAT-DELETION (at least on instances with non-mixed clauses, satisfying (P1)-(P3)) would improve on currently the best polynomial time approximation factor $\left(2-\frac{\log \log n}{2 \log n}\right)$ for the MiN-VC problem.

Theorem 4 and its proof can be modified also to situations when the quality of approximation of the algorithm $\mathscr{A}$ for Min-2SAT-DELETION is measured in different parameters. Consider an algorithm $\mathscr{A}$ for Min-2SAT-DeLETion that is robust on almost-satisfiable instances. That means, for an instance $\mathcal{F}$ of MiN2SAT-DELETION, whose optimum assignment leaves only $\varepsilon$ fraction of clauses unsatisfied, $\mathscr{A}$ finds an assignment that leaves at most $g(\varepsilon)$ fraction of clauses of $\mathcal{F}$ unsatisfied, where $g:\langle 0,1\rangle \rightarrow\langle 0,1\rangle$ is a function with $\lim _{\varepsilon \rightarrow 0} g(\varepsilon)=g(0)=0$.

Zwick's efficient algorithm [15] has this robustness property with $g(\varepsilon)=$ $5 \varepsilon^{1 / 3}$. An interesting question is whether such algorithms exist with $g(\varepsilon)=$ $o\left(\varepsilon^{1 / 2}\right)$, and if yes, how far one can go beyond this bound. One can easily check (along the lines of the proof of Theorem 4) that any robust algorithm with $g(\varepsilon)=O\left(\varepsilon^{t}\right), t \in(0,1\rangle$, gives us an $\left(2-c \cdot(1+\bar{d})^{1-1 / t}\right)$-approximation algorithm for Min-VC-PM on graphs with average degree $\bar{d}:=\frac{2|E|}{|V|}$ (here $c \in(0,1)$ is an absolute constant). For any $t>\frac{1}{2}$ the existence of a robust algorithm with $g(\varepsilon)=O\left(\varepsilon^{t}\right)$ would significantly improve (for large $\bar{d}$ ) on currently the best approximation factor $\left(2-\frac{5}{2 \bar{d}+3}\right)$ on polynomial time approximation for MINVC-PM on graphs with average degree $\bar{d}[7]$.

\subsection{Bounded Occurrence Instances of 2SAT Problems}

One can obtain inapproximability results also for bounded occurrence instances of Min-2SAT-Deletion using inapproximability results for Min-VC on bounded degree graphs. We will show that on an example of cubic graphs. One can check that instances produced in [4] to achieve the inapproximability results for the Min-VC problem in cubic graphs, have a perfect matching. It is proven there that it is NP-hard for a cubic graph $G$ with $n$ vertices and having a perfect matching to distinguish the case $v c(G)<\left(\frac{1}{2}+2 \delta+\varepsilon\right) n$ from the case $v c(G)>\left(\frac{1}{2}+3 \delta-\varepsilon\right) n$ where $\delta>0$ is a positive constant related to parameters of an amplifier used in the construction, and $\varepsilon \in\left(0, \frac{\delta}{2}\right)$ can be arbitrarily small independently of $\delta$. The instance $\mathcal{F}(G, M)$ that corresponds to such $G$ (and an arbitrary matching $M$ in $G$ ) has $n$ variables, $2 n$ clauses (all non-mixed and satisfying (P1)-(P3)) with exactly 4 occurrences of each variable. Due to Lemma 2, the corresponding NP-hard problem is to distinguish $\operatorname{OPT}(\mathcal{F}(G, M))<(2 \delta+\varepsilon) n$ from $\operatorname{OPT}(\mathcal{F}(G, M))>(3 \delta-\varepsilon) n$. We have just proved

Theorem 5. It is NP-hard to approximate the Min-2SAT-DeLETION problem to within any constant approximation factor smaller than $\frac{3}{2}$ on instances with 
exactly 4 occurrences of each variable, no mixed clauses, and satisfying conditions (P1)-(P3).

In this way one can derive inapproximability results also for the complementary MAX-2SAT satisfiability problem with exactly four occurrence of each variable. The corresponding NP-hard problem is to decide whether the optimum is greater than $(2-2 \delta-\varepsilon) n$, or less than $(2-3 \delta+\varepsilon) n$. Now the inapproximability factor $\approx \frac{2-2 \delta}{2-3 \delta}$ depends crucially on parameters of an amplifier (hidden in $\delta$ ) used in that hardness result for cubic graphs. From estimates of [4], the inapproximability to within $1+\frac{1}{385}$ follows. It is worse than the recent hardness factor $1+\frac{1}{268}$ obtained for this problem in [2], but on the other hand, it applies to instances with no mixed clauses.

\section{Concluding Remarks}

It has been conjectured by several authors that it is NP-hard to approximate Min-VC within any constant factor smaller than 2. By Theorem 4 and Corollary 1 this would imply also NP-hardness of approximating Min-2SAT-DeLETION within any constant factor. We think that the latter problem is significantly easier than the former one. The methods used in this paper show that in order to prove NP-hardness to approximate Min-2SAT-DeLETION within (any fixed constant) $k$, it suffices to provide instances $G=(V, E)$ with a perfect matching for which it is NP-hard to distinguish $v c(G)<\frac{1}{2}|V|+\varepsilon(G)$ from $v c(G)>\frac{1}{2}|V|+k \cdot \varepsilon(G)$, for some efficiently computable function $\varepsilon=\varepsilon(G)>0$. On the other hand, the problem Min-VC requires to show, for arbitrarily small constant $\epsilon>0$, NP-hardness to distinguish instances with $v c(G)<\left(\frac{1}{2}+\varepsilon\right)|V|$ from those with $v c(G)>(1-\varepsilon)|V|$.

\section{References}

1. R. Bar-Yehuda and S. Even, A local-ratio theorem for approximating the weighted vertex cover problem, Annals of Discrete Mathematics 25(1985), $27-46$.

2. P. Berman and M. Karpinski, Improved approximation lower bounds on small occurence optimization, ECCC Report TR03-08, 2003.

3. J. Chen and I. A. Kanj, On approximating minimum vertex cover for graphs with perfect matching, Proc. of the 11st ISAAC, Taipei, Taiwan, LNCS 1969, Springer, 2000, pp. 132-143.

4. M. Chlebík and J. Chlebíková, Inapproximability results for bounded variants of optimization problems, Proc. of the 14th FCT, LNCS 2751, Springer Verlag, 2003, pp. 27-38, also ECCC Report TR03-26, 2003.

5. I. Dinur and S. Safra, The importance of being biased, STOC 2002, pp. 33-42, also ECCC Report TR01-104, 2001.

6. J. Håstad, Some optimal inapproximability results, Journal of ACM 48(2001), 798-859. 
7. M. Halldórsson and J. Radhakrishnan, Greed is good: approximating independent sets in sparse and bounded-degree graphs, Algorithmica 18 (1997), $145-163$.

8. S. Khanna, M. Sudan and L. Trevisan, Constraint Satisfaction: The approximability of minimization problems, Proceedings of the 12th Annual IEEE Conference on Computational Complexity, Ulm, Germany, 24-27 June, 1997, pp. 282-296.

9. P. Klein, S. Plotkin, S. Rao and E. Tardos, Approximation algorithms for Steiner and directed multicuts, Journal of Algorithms 22(2) (1997), 241-269.

10. C. H. Little, D. D. Grant and D. A. Holton, On defect $d$ matchings in graphs, Discrete Mathematics 13(1975), 41-54. Erratum in Disc. Math. 14(1976), 203.

11. M. Lewin, D. Livnat and U. Zwick, Improved rounding techniques for the MAX-2SAT and MAX Di CuT problems, IPCO 2002, LNCS 2337, pp. 67-82.

12. B. Monien and E. Speckenmeyer, Ramsey numbers and an approximation algorithm for the vertex cover problem, Acta Informatica 22(1985), 115-123.

13. G. L. Nemhauser and L. E. Trotter, Vertex packings: structural properties and algorithms, Math. Programming 8(1975), 232-248.

14. D. B. West, Introduction to graph theory, Prentice-Hall, 2001.

15. U. Zwick, Finding almost satisfying assignments, Proc. of the 30th Annual ACM Symposium on Theory of Computing, 1998, pp. 551-560. 